\section{Abriter les sans-abri}

$\mathrm{E}$ n août 2006, l'annonce d'une réduction du financement fédéral des programmes pour les sans-abri ${ }^{1}$ a soulevé des préoccupations. Les défenseurs des sansabri craignent toujours que le gouvernement actuel n'ait l'intention d'éliminer les programmes fédéraux de lutte contre l'itinérance, même si la ministre fédérale des Ressources humaines et du Développement social, Diane Finley, s'est empressée de le nier. La disparition du leadership fédéral dans la lutte contre l'itinérance aurait un effet négatif non seulement sur les refuges pour itinérants, mais aussi sur les hôpitaux et le système de santé. On croit que les investissements à long terme dans des logements abordables et subventionnés, les services de santé mentale et le soutien du revenu nécessaires pour mettre fin à l'itinérance au Canada seront inintéressants pour un gouvernement qui tente de se positionner pour remporter une majorité.

Sur le plan historique, les refuges pour itinérants remontent à un modèle de charité qui consistait à secourir les personnes en situation de crise temporaire. À la suite de décennies de modifications des politiques sociales et de santé, conjuguées au déclin des systèmes de soutien familiaux et communautaires, des milliers des Canadiens les plus vulnérables doivent se tourner vers les refuges d'urgence et services connexes pour survivre. Même s'il est vrai que beaucoup d'itinérants qui se présentent à un refuge ont besoin de services à court terme, il reste que dans tout milieu urbain, au moins 10 \% de ces personnes sont sans abri depuis plus d'un an. Cette population est la plus vulnérable à la dégradation des services aux itinérants et c'est elle que l'on associe aux coûts élevés des soins de santé et de l'appareil judiciaire en l'absence de systèmes de remplacement. Notre filet de sécurité sociale, qui doit protéger les populations vulnérables, laisse de plus en plus tomber ceux et celles dont les besoins sont les plus grands, ce qui entraîne des souffrances humaines et des coûts inutiles pour le secteur de la santé et des services sociaux et pour l'appareil judiciaire.

Il fut un temps où le système de santé offrait un filet à toute épreuve à cette population. Ceux et celles qui ne pouvaient vivre avec l'aide disponible dans la communauté vivaient dans des établissements. On a jugé ce modèle trop coûteux pour les contribuables et il est indéniable qu'il empêchait l'individu de jouir des droits fondamentaux accordés à tous les citoyens. Les établissements répondaient toutefois à certains des besoins de cette population, assuraient sa sécurité et géraient les coûts des soins dispensés. Malheureusement, la vie dans un refuge sans soutien approprié est associée à des * coûts élevés en services inefficaces aussi bien judiciaires que de santé d'urgence, qui ne procurent que peu d'avantages à la société ou à la personne concernée. Nous avons accepté un modèle d'inefficacité des coûts et d'isolement social au lieu de prendre la responsabilité des logements abordables et des services nécessaires pour permettre à nos citoyens les plus vulnérables de vivre dans la dignitée,
Les promoteurs des soins de santé qui travaillent auprès des sans-abri sont frustrés par le fait que la politique gouvernementale ne tient pas compte des preuves au sujet de l'efficacité. En dépit de la masse de données démontrant qu'un éventail de stratégies d'atténuation du préjudice sauve des vies et évite des coûts en soins de santé, d'autres arguments et orientations continuent d'influencer la politique gouvernementale. Les politiques selon lesquelles un groupe vulnérable (les toxicomanes, par exemple) n'est pas digne de certains aspects des soins mettent en danger tous nos groupes vulnérables (par exemple, les immigrants, les personnes âgées, les personnes qui souffrent d'un retard du développement). Même si les données démontrent qu'un logement de soutien abordable assorti des soins de santé de longue durée appropriés constitue un moyen efficace de réduire l'itinérance dans les populations vulnérables, les gouvernements hésitent à y consacrer les ressources nécessaires. Les solutions prévoyant l'accès à court terme à un logement comportant des services de santé mentale et autres ainsi que des possibilités de logement sur le marché privé sont peutêtre populaires sur le plan politique, mais les données indiquent qu'elles sont inefficaces ${ }^{4}$. La politique sociale factuelle va malheureusement à l'encontre de ce qui est plus acceptable sur le plan politique. Le secteur caritatif ne peut ni ne doit accepter l'entière responsabilité des coûts pour s'occuper des personnes de notre société qui ont les besoins les plus complexes. Les contributions du secteur caritatif dans le domaine de l'itinérance sont importantes et précieuses, mais elles ne peuvent remplacer des politiques fondées sur des données scientifiques. On ne doit pas s'attendre à ce que les groupes de bienfaisance formulent et mettent en œuvre une politique nationale intégrée sur l'itinérance. Ce rôle incombe au gouvernement et à nos représentants élus.

\section{Wendy Muckle \\ Ottawa Inner City Health, Inc. \\ Jeffrey Turnbull \\ Département de médecine \\ Université d'Ottawa \\ Ottawa (Ontario)}

Cet article a fait l'objet d'un examen par les pairs.

\section{RÉFÉRENCES}

I. Déclaration de l'honorable Diane Finley, ministre des Ressources humaines et du Développement social [communiqué]. Ottawa, le I7 août 2006. Disponible : http:// news.gc.ca/cfmx/view/fr/index.jsp?articleid=233559 (consulté le I2 octobre 2006).

2. Steve Pomeroy Focus Consulting Inc. Invest or subsidize: comparative subsidy cost of non-profit and private market units rent supplements in Ottawa 1978-2004. Mai 2005. Disponible : www.onpha.on.ca/affordable_housing_initiatives/fight_resources /doc/ottawa_final.pdf (consulté le 6 octobre 2006).

3. Plumb JD. Homelessness: reducing health disparities [éditorial]. JAMC 2000;163:172-3.

4. Kushel MB, Vittinghoff E, Hass JS. Factors associated with the health care utilization of homeless persons. JAMA 2001;285:200-6. 